\title{
Editorial
}

\section{Progress in, and future prospects for, the treatment of primary pulmonary hypertension}

This is an exciting time to be in pulmonary hypertension research. The past few years have witnessed a steady flow of publications documenting the beneficial effects of prostacyclin treatment in primary pulmonary hypertension $(\mathrm{PPH})$ and other forms of pulmonary arterial hypertension and the emergence of new drugs with therapeutic possibilities in these conditions. More recently, genetic analysis of familial PPH has identified a molecular mechanism that opens up new opportunities for pharmacological intervention in this disease.

Untreated $\mathrm{PPH}$ has an appalling prognosis, with a median survival of 2.5 years. Both warfarin and calcium antagonists (for those who can tolerate them) improve survival but the most significant development in the treatment of PPH to date has been the use of intravenous prostacyclin and prostacylin analogues. Introduced initially as a bridge to transplantation, the shortage of suitable donors meant that many patients remained on treatment for several years. Controlled clinical trials have demonstrated that prostacyclin infusion improves exercise tolerance and pulmonary haemodynamics in both $\mathrm{PPH}^{1}$ and pulmonary hypertension secondary to scleroderma. ${ }^{2}$ Indeed, prostacyclin treatment may now be considered superior to lung transplantation in improving the quality of life and survival in such patients. ${ }^{3}$ Furthermore, the benefit of this treatment is also observed in patients with no acute vasodilator response to the drug, suggesting that in addition to vasorelaxation and its antiplatelet effects, prostacyclin may inhibit vascular remodelling. ${ }^{4}$ Although inhibition or reversal of vascular remodelling remains to be demonstrated clinically, studies with human pulmonary smooth muscle cells in vitro show that prostacyclin analogues inhibit growth, and that this effect is most evident in cells derived from the distal pulmonary vascular bed. ${ }^{5}$

Intravenous prostacyclin is now established as a treatment to be considered for all patients with PPH but its use is frought with difficulties. Aside from the constant risk of infection from chronic vascular access, it is associated with vomiting, systemic hypotension, dizziness, syncope, flushing, and headache. Tachyphylaxis develops with continued use, necessitating regular dose escalations. Once started, it cannot be discontinued. This has led to studies of alternative methods of drug delivery. One approach is nebulised iloprost. ${ }^{6}$ The local delivery of the drug to the pulmonary vascular bed has attractions in terms of reducing systemic side effects, but its short half life necessitates three hourly administration, each episode lasting 15 minutes. Another option is subcutaneous infusion of a prostacyclin analogue (uniprost) using a portable minipump, ${ }^{7}$ but some patients develop intolerable pain at the injection site.

\section{Oral prostacyclin}

Oral administration has been limited by poor bioavailability, but stable analogues are now available and have been the subject of small clinical studies. One such analogue, beraprost, reduces pulmonary artery pressure acutely ${ }^{8}$ and the haemodynamic benefits appear to be maintained long term and to be associated with a reduction in mortality as well as pulmonary artery pressure. ${ }^{9}$ The most recent study, published in this issue, reports an increase in exercise tolerance ${ }^{10}$ However, these studies are uncontrolled and the assessments have been made against historical data. Larger controlled studies are needed to evaluate accurately the role of oral prostacyclin analogues.

Oral prostacyclin analogues may extend the benefits of prostacyclin treatment to a greater proportion of patients with PPH, but there is still considerable room for improvement in quality of life. The effect of prostacyclin treatment on exercise tolerance (the six minute walk test) varies from inhibition of deterioration (compared with a placebo group) to up to a $30 \%$ improvement on the pre-treatment baseline, and side effects are common. There is a need for more effective orally active medication that acts preferentially on the pulmonary vasculature (as opposed to both pulmonary and systemic vessels) if further improvements are to be made while minimising the risk of side effects.

\section{New treatments}

Investigation of the pathobiology of pulmonary arterial hypertension has suggested alternative treatments. Most advanced in this respect are the endothelin antagonists. Endothelin (ET) is a potent vasoconstrictor and a mitogen. In vitro and in vivo data support a role for ETs in structural remodelling and the progression of the disease. Bosentan, an antagonist of both $\mathrm{ET}_{\mathrm{A}}$ and $\mathrm{ET}_{\mathrm{B}}$ receptor subtypes, has been shown to reduce pulmonary artery pressure acutely in patients with pulmonary hypertension. ${ }^{11}$ It has recently completed trials in a randomised placebo controlled study in patients with PPH and pulmonary hypertension associated with scleroderma, where the drug was given for three months before starting prostacyclin. Early reports ${ }^{12}$ indicate that bosentan increased exercise tolerance to a degree commensurate with that reported with prostacyclin infusion at a similar stage in the illness, and a licence for this drug in PPH seems likely. Other endothelin antagonists are also under investigation in $\mathrm{PPH}$. There are theoretical reasons suggesting a selective $\mathrm{ET}_{\mathrm{A}}$ may have advantages over a combined $\mathrm{ET}_{\mathrm{A}} / \mathrm{ET}_{\mathrm{B}}$ antagonist; $\mathrm{ET}_{\mathrm{A}}$ receptors mediate vasoconstriction and growth while $\mathrm{ET}_{\mathrm{B}}$ receptors on endothelial cells mediate nitric oxide and prostacyclin release. It remains to be shown whether this difference in receptor function can be exploited for therapeutic benefit in vivo.

Of equal interest are the phosphodiesterase (PDE) inhibitors, particularly those directed at PDE type 5, such as sildenafil. The lung is rich in PDE5, which metabolises and limits the activity of cyclic GMP. This cyclic nucleotide mediates the vascular actions (vasorelaxation and growth inhibition) of nitric oxide and natriuretic peptides, and inhibition of PDE5 offers a therapeutic approach to enhancing the effects of these vasoactive factors. Studies in 
animals have shown that PDE5 inhibition greatly attenuates hypoxia induced pulmonary hypertension and attendant vascular remodelling when treatment is started before exposure to hypoxia. Interestingly, mice genetically deficient in endothelial nitric oxide synthase also show some response to the drug, indicating that other biochemical stimulants of cyclic guanosine monophosphate (GMP) (for example, natriuretic peptides) contribute to the beneficial effect. In humans, pretreatment with a single oral dose of sildenafil $100 \mathrm{mg}$ inhibits the pressor response to $11 \%$ oxygen in healthy volunteers. ${ }^{13}$ There have been isolated case reports where oral sildenafil has been used as an alternative to prostacyclin in PPH with early success. ${ }^{14} 15$ Further evaluation of sildenafil in $\mathrm{PPH}$ patients is now warranted.

\section{New discoveries}

Recent studies of familial cases of PPH have provided a more fundamental insight into the pathology of the condition. Analysis of genes within a region (PPH1 locus) on chromosome $2 \mathrm{q} 33$ that had been shown to segregate with $\mathrm{PPH}$ led to the identification of a series of mutations in the $B M P R 2$ gene. ${ }^{16}{ }^{17}$ This gene encodes the type II bone morphogenetic receptor (BMPR-II), a member of the TGF- $\beta$ superfamily, and mutations have been found in up to $60 \%$ of patients with a family history of clinical disease and $25 \%$ of so-called sporadic cases. The receptor binds bone morphogenetic proteins (BMPs) types 2,4 , and 7 as well as other ligands and, through dimerisation with a type I receptor, initiates intracellular signalling via several pathways, most notably transcription factors known as Smads. ${ }^{18}$ These transcription factors translocate to the nucleus and regulate gene transcription. Experiments with pulmonary vascular smooth muscle cells in culture indicate that BMPs inhibit growth. The mutations identified in $B M P R 2$ would be expected to disrupt ligand binding and/or receptor signalling, impair BMPR-II function, and remove a mechanism for keeping vascular remodelling in check.

The functional consequences of BMPR2 mutations need to be tested in an experimental system. An experimental model of PPH would be very helpful for investigating the pathogenesis of the disease and evaluating new treatments but may be difficult to engineer. It is clear that loss of one $B M P R 2$ allele is not in itself sufficient to cause the phenotype. Not only does the disease not manifest itself until the third or fourth decade, people with $B M P R 2$ mutations have been identified who have no evidence of pulmonary hypertension. It is likely that a second insult is required before the phenotype is expressed.

The view is emerging that $\mathrm{PPH}$ is caused by an insult to the pulmonary vasculature in a genetically predisposed individual, and that factors such as impaired nitric oxide and prostacyclin production and increased endothelin activity are mediators of disease progression. There will remain a role for drugs that interfere to correct such perturbations for some time to come. Current studies have naturally turned to the effect of prostacyclin analogues, endothelin antagonists, and PDE5 inhibitors on BMPR-II activity and will provide a better rational for their use as well as clues to new therapeutic approaches. In time to come we look forward to the correction of BMPR2 gene mutations or some component of its signalling pathway by gene therapy. However, in the immediate future, the use of combination oral therapy based on small molecules is likely to be the basis of treatment for PPH and related diseases.

MARTIN R WILKINS JOHN WHARTON

Section on Clinical Pharmacology,

Faculty of Medicine,

Imperial College,

Hammersmith Hospital,

Du Cane Road,

London W12 NN, UK

m.wilkins@ic.ac.uk

1 Barst RJ, Rubin LJ, Long WA, et al. A comparison of continuous intravenous epoprostenol (prostacyclin) with conventional therapy for primary pulmonary hypertension. $N$ Engl F Med 1996;334:296-301.

2 Badesch DB, Tapson VF, McGoon MD, et al. Continuous intravenous epoprostenol for pulmonary hypertension due to the scleroderma spectrum of prostenol for pulmonary hypertension due to the scleroderma spectrum of

3 Higenbottam T, Siddons T. Trials of inhaled lloprost and other new vasodilating prostaglandins. Eur Respir f 2001;17:6-7.

4 McLaughlin VV, Genthner D, Panella M, et al. Reduction in pulmonary vascular resistance with long-term epoprostenol (prostacyclin) therapy in primary pulmonary hypertension. N Engl f Med 1998;338:273-7.

5 Wharton J, Davie N, Upton P, et al. Prostacyclin analogues differentially inhibit growth of distal and proximal human pulmonary artery smooth muscle cells. Circulation 2000;102:3130-6.

6 Hoeper MM, Schwarze M, Ehlerding S, et al. Long-term treatment of primary pulmonary hypertension with aerosolized iloprost, a prostacyclin analogue. N Engl f Med 2000;342:1866-70.

7 Barst RJ, Simonneau G, Rich S, et al. Efficacy and safety of chronic subcutaneous infusion UT-15 in pulmonary arterial hypertension (PAH) subcutaneous infusion UT-15 in pulmonary arter

8 Saji T, Ozawa Y, Ishikita T, et al. Short-term hemodynamic effect of a new oral PGI2 analogue, beraprost, in primary and secondary pulmonary hypertension. Am $\mathcal{F}$ Cardiol 1996;78:244-7

9 Nagaya N, Uematsu M, Okano Y, et al. Effect of orally active prostacyclin analogue on survival of outpatients with primary pulmonary hypertension. f Am Coll Cardiol 1999;34:1188-92.

10 Vizza CD, Sciomer S, Morelli S, et al. Long term treatment of pulmonary arterial hypertension with beraprost, an oral prostacyclin analogue. Heart 2001;86:661-5.

11 Williamson DJ, Wallman LL, Jones R, et al. Hemodynamic effects of bosentan, an endothelin receptor antagonist, in patients with pulmonary hypertension. Circulation 2000;102:411-18.

12 Channick RN, Rubin LJ, Simonneau G, et al. Bosentan, a dual endothelin receptor antagonist, improves exercise capacity and haemodynamics in patients with pulmonary arterial hypertension: results of a double blind, randomized, placebo-controlled trial. Circulation 2000;102(suppl II).

13 Zhao L, Mason NA, Morrell NW, et al. Sildenafil inhibits hypoxia-induced pulmonary hypertension. Circulation 2001;104:424-8.

14 Abrams D, Schulze-Neick I, Magee AG. Sildenafil as a selective pulmonary vasodilator in childhood primary pulmonary hypertension. Heart 2000;84: e4.

15 Prasad S, Wilkinson J, Gatzoulis MA. Sildenafil in primary pulmonary hypertension. New Engl f Med 2000;343:1342-3.

16 Deng Z, Morse JH, Slager SL, et al. Familial primary pulmonary hypertension (gene PPH1) is caused by mutations in the bone morphogenetic protein receptor-II gene. Am f Hum Genet 2000;67:737-44.

17 The International PPH Consortium. Heterozygous germline mutations in $B M P R 2$, encoding a TGF- $\beta$ receptor, cause familial primary pulmonary hypertension. Nature Genetics 2000;26:81-4.

18 Massague J, Chen YG. Controlling TGF-beta signaling. Genes Dev $2000 ; 14: 627-44$ 